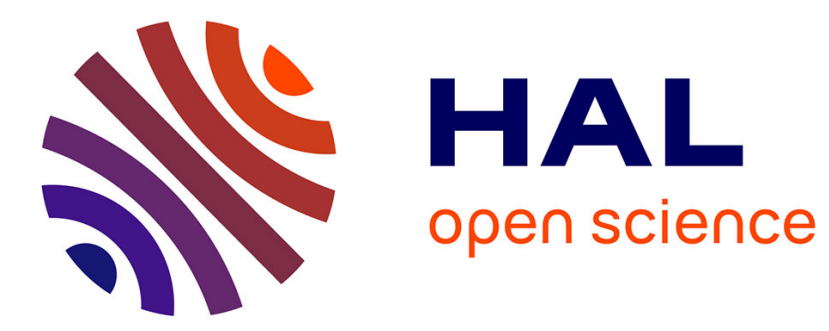

\title{
Fonctionnement des cadres spatiaux dans les résumés de films
}

\author{
Laure Sarda
}

\section{To cite this version:}

Laure Sarda. Fonctionnement des cadres spatiaux dans les résumés de films. Langue française, 2005, 148: Les Adverbiaux Cadratifs, pp.61-79. halshs-00067816

\section{HAL Id: halshs-00067816 https://shs.hal.science/halshs-00067816}

Submitted on 9 May 2006

HAL is a multi-disciplinary open access archive for the deposit and dissemination of scientific research documents, whether they are published or not. The documents may come from teaching and research institutions in France or abroad, or from public or private research centers.
L'archive ouverte pluridisciplinaire HAL, est destinée au dépôt et à la diffusion de documents scientifiques de niveau recherche, publiés ou non, émanant des établissements d'enseignement et de recherche français ou étrangers, des laboratoires publics ou privés. 


\title{
Fonctionnement des cadres spatiaux dans les résumés de films »
}

\author{
Laure Sarda
}

\section{LATTICE UMR 8094 CNRS \& ENS \\ laure.sarda@ens.fr}

Cet article $^{1}$ aborde la description des adverbiaux spatiaux en position détachée initiale dans le cadre du Modèle de l'Encadrement du Discours (Charolles 1997). La première partie expose ce modèle et décrit le fonctionnement 'cadratif' des adverbiaux spatiaux en tête de phrase. La deuxième partie présente le corpus des résumés de films sur lequel repose cette étude. Nous nous concentrons ensuite sur deux questions spécifiques, celle du repérage des fins de portée (scope) des cadres spatiaux ( $3^{\text {ème }}$ partie) et celle de leur fonctionnement comme ancrage du discours ( $4^{\text {ème }}$ partie).

\section{Averbiaux cadratifs}

Charolles (1997) définit les cadres de discours, en s'appuyant sur la notion d'univers de discours de R. Martin (1983). Un cadre est une unité textuelle regroupant des propositions qui entretiennent un même rapport avec un critère sémantique d'interprétation spécifié par un introducteur de cadre: on peut trouver des introducteurs de cadre d'ordre thématique, organisationnel, énonciatif, temporel ou spatial respectivement illustré par les exemples (1-5)

(1) En ce qui concerne les sciences, les étudiants sont moins nombreux.

(2) Premièrement, les étudiants sont moins nombreux. Deuxièmement, ils travaillent mieux.

(3) Selon Marie, Paul est malade.

(4) Hier, Paul est tombé malade.

(5) A Paris, Paul est malade.

Nous rappelons ici les principales propriétés des introducteurs de cadres :

Une expression introductrice de cadre (EIC) cumule fonction représentationnelle et fonction instructionnelle ou procédurale du fait qu'elle indexe, non seulement la proposition à l'initiale de laquelle elle apparaît, mais aussi, potentiellement, un certain nombre de propositions subséquentes.

(6) Dans le sud de l'Espagne,(P1) les amandes sont mûres au mois d'octobre. (P2) Les figues arrivent à maturité même tardivement dans les montagnes. A Paris, (P3) si les figues ne sont pas mûres en septembre, elles ne mûrissent pas. (P4) Elles pourrissent avec l'arrivée du froid et de la pluie.

Dans cet exemple, l'adverbial en tête de phrase (dans le sud de l'Espagne) étend sa portée jusqu'à l'apparition d'un second adverbial en tête (A Paris) qui, lui-même, porte non seulement sur P3 mais aussi sur P4.

Cette fonction d'indexation est considérée comme une marque de cohésion discursive à côté d'autres marques de cohésion plus classiques que sont les anaphores (relations référentielles) et les connecteurs (relations rhétoriques), regroupés sous l'étiquette des marques de connexion (cf. Halliday \& Hassan 1976, Reinhart 1981; Sanders \& Spooren 2001). Dans Charolles (2003) et Charolles \& alii (2005) connexion et indexation sont définies comme deux familles dans la typologie générale des marques de cohésion. La connexion implique des relations remontantes,

\footnotetext{
${ }^{1}$ Je remercie Laurence Delort pour sa relecture attentive et ses commentaires judicieux.
} 
qui ont une incidence sur le topique de discours local et qui sont hiérarchiques. L'indexation implique au contraire des relations descendantes, non topicalisantes et non hiérarchiques (dans la mesure où les informations sont listées). Une propriété supplémentaire découle de ces dernières : l'indexation assume non seulement une fonction de cohésion en regroupant plusieurs propositions sous un même index, mais aussi une fonction de segmentation en découpant des blocs dans le discours. Enfin, cette fonction d'indexation est attachée à la position initiale préverbale. La capacité des adverbiaux à étendre leur portée au-delà de leur phrase d'accueil ne vaut, en effet, que s'ils apparaissent en tête de celle-ci.

Parmi ces propriétés, celle qui touche au rôle topicalisant ou non topicalisant des adverbiaux soulève un débat important (Charolles 2003, Prévost 2003, Vieu \& alii 2005). Le statut potentiellement topical des EIC est en effet une question délicate sur laquelle nous nous attarderons un instant avant de la reprendre dans la quatrième partie. Charolles écrit (Charolles à par.) :

"Les expressions cadratives ne sont pas thématiques (topicales), dans le sens où, en principe, elles ne signalent pas ce sur quoi porte le segment en tête duquel elles sont détachées (ce ne sont pas des "topics of discourse »). Elles fournissent des indications procédurales destinées à faciliter la subdivision des informations textuelles et leur récupération en mémoire (comme dans les tests de mémoire où, pour récupérer une information, on l'associe à un lieu connu) ».

Mais dans (Charolles 2003), l'auteur reprends cette question en détail et nuance son propos. L'idée avancée est la suivante.

... « il n'est pas possible de statuer sur l'à propos (et donc l'adressage) des circonstants antéposés à partir du seul contenu de la proposition les accueillant. Il faut tenir compte du topique de discours, lequel varie au fur et à mesure que celui-ci avance. (p.41).

Les propositions qui tombent sous leur portée [des adverbiaux cadratifs] ne sont au sujet de l'index qu'ils mettent en avant que lorsque le discours ne fournit aucun autre topique à même de remplir cette fonction. Le fait que cette possibilité existe oblige à considérer qu'il n'est pas possible de statuer sur le topique (aboutness) d'une phrase isolée (p. 44)».

Les exemples discutés par Charolles sont reportés en (7) et (8)

(7) Lola sortit faire un tour. En bas de l'immeuble, elle croisa le facteur. Il portait un énorme sac et elle lui proposa de l'aider.

(8) Lola sortit faire un tour. En bas de l'immeuble, un homme faisait les cents pas. Une fine couche de givre recouvrait le sol. Des enfants rentraient de l'école en se chamaillant.

Dans l'exemple repris en (7), l'auteur affirme que « le circonstant spatial se contente de fixer le cadre où se déroule une série d'événements qui ont une cohérence indépendamment de l'endroit où ils se produisent. Ce cadre sert d'arrière plan, il n'est pas le topique adressé du commentaire, ni des phrases qui suivent». (...) En revanche, dans l'exemple repris sous (8), «le texte est à propos de ce qui se passe dans le lieu indiqué par le SP antéposé, les événements n'ont d'autre point commun que de se dérouler dans le même endroit et cela suffit pour conférer au passage une cohérence a minima » (p. 40).

Nous voyons que la fonction d'une même expression peut varier selon la suite du discours. Il est donc primordial d'en tenir compte.

Dans le même volume (Charolles \& Prévost 2003), l'article de Prévost amène à considérer les notions de topique et de cadre dans la continuité l'une de l'autre. L'auteur examine une série d'exemples qui « militent en faveur du rapprochement, voire de l'assimilation, entre un certain type de topique, ceux qui sont détachés et non liés, et un certain type de cadre, ceux qui limitent d'un point de vue notionnel l'applicabilité de la prédication qui suit » (p. 55), rapprochant ainsi 
des énoncés comme Paul, je n'ai toujours pas de nouvelles et Quant à Paul, je n'ai toujours pas de nouvelles. L'auteur se place dans la perspective d'un continuum qui partirait « du topique par excellence $(9)^{2}$ puis traverserait une zone mixte (10-11), avant d'arriver aux adverbiaux spatiotemporels dont le caractère cadratif est le plus souvent bien net $\gg(12)$.

(9) Tu as des nouvelles de Paul? - Oui, il m'a téléphoné hier.

(10) Paul, ses parents sont vraiment adorables.

Paul, je n'ai toujours pas de nouvelles.

(11) Quant à Paul, il m'a téléphoné hier.

Quant à Paul, je n'ai toujours pas de nouvelles.

(12) Ce matin, j'ai rencontré Marie. Elle n'a pas l'air très en forme.

Sur le bassin d'Arcachon, on continue à trouver des galettes de fuel. Il en revient à chaque grande marée.

Ce classement et le traitement spécial des adverbiaux spatio-temporels, dont l'auteur souligne le caractère nettement cadratif est mis en relation avec deux types de topiques qu'envisage Chafe (1976) : ceux qui établissent un cadre notionnel («individual framework»), et ceux qui établissent un cadre spatio-temporel; la notion d' «aboutness » serait applicable au premier mais pas au second.

L'auteur propose, en outre, de traiter différemment ces deux types de cadres notionnel et spatiotemporel, les uns intervenant au niveau de l'énonciation, les autres au niveau de l'énoncé. Dans les exemples 10-11, on aurait donc affaire «à un cadre énonciatif (d'ordre notionnel/ thématique), par rapport auquel il est pertinent d'énoncer la relation prédicative qui suit» (p.57). Alors que dans les exemples sous 12, «il s'agit d'un cadre pour l'énoncé dans lequel la relation prédicative qui suit est pertinente ${ }^{3} »$ (id.). Le continuum entre topique et cadre est ainsi formulé :

(13) Topique intégré $\rightarrow$ topique non intégré $\rightarrow(\mathrm{ou}=)$ cadre énonciation $\rightarrow$ cadre énoncé

Ce continuum cantonne les adverbiaux spatiaux temporels à la seule fonction cadrative, à l'écart des positions mixtes en cadre et topique. Mais, l'auteur ne s'en tient pas là et reconnaît, avec (Charolles 2003) que les cadres spatio-temporels peuvent tomber sous le coup d'un phénomène de « topicalisation contextuelle» en fonction de la suite du discours. Cependant, dans la mesure où c'est le contexte qui permet de résoudre la double interprétation (cadre ou topique), l'auteur assimile ce cas de figure à un phénomène d'ambiguïté. Elle examine en revanche des énoncés comme sur le pont d'Avignon, on y danse... où la préposition introduisant l'adverbial fait l'objet d'une reprise pronominale. Dans cette forme d'énoncé, l'adverbial est construit a posteriori par la reprise pronominale comme un pré-topique. S'il s'agit d'un complément argumental comme dans $A$ Paris, $j^{\prime} y$ vais, alors l'auteur suggère que nous avons affaire à un focus (déplacé en tête). Dans ces deux cas, c'est l'effet de redondance de la reprise pronominale qui invite à reconsidérer le rôle informationnel des adverbiaux pour leur attribuer le statut soit de topique, soit de focus.

Dans la partie 4, nous décrirons comment certaines formes de détachement peuvent créer un « arrière-plan » servant à situer de manière diffuse une série d'événements, avec lesquels ils ne sont pas coïndicés et dont ils ne constituent pas l'à propos. Pour l'heure, nous poursuivons par la présentation du corpus (\$2) avant d'aborder la question de la portée des adverbiaux (§3), question qui, à notre avis, ne peut être abordée complètement indépendamment du débat dont nous venons de faire état sur le statut informationnel des adverbiaux.

\footnotetext{
${ }^{2}$ Nous reportons les exemples que l'auteur propose p. 55-56.

${ }^{3}$ L'auteur conçoit la pertinence dans le sens où le topique est défini comme une relation d'aboutness (relation pragmatique de pertinence).
} 


\section{Présentation du corpus}

Notre corpus est constitué de 65 « résumés de films ». 27 d'entre eux sont tirés de programmes télé. Le reste a été glané sur Internet, à savoir 32 résumés des épisodes de X-Files ${ }^{4}$, plus 5 résumés supplémentaires dont quatre d'un même film (la cérémonie). Nous n'entrerons pas dans le débat de savoir si le résumé de film constitue un genre. Il est évident que cette question mériterait d'être approfondie par des études extensives qui cependant, nous éloigneraient de notre objet. Nous nous contentons de constater un certain nombre de caractéristiques propres aux textes retenus ici: il s'agit de textes courts (5-6 lignes en moyenne), monoblocs, majoritairement entièrement au présent. Ils introduisent des référents spatiaux, distribués pour un certain nombre en tête de phrase (14), mais pas uniquement. Plus précisément, 23 textes commencent par un adverbial spatial, 13 par un adverbial non-spatial (le plus souvent temporel). Lorsque l'espace n'est pas introduit en début de texte, il apparaît en position intra-prédicative, dans la première proposition (15), et/ou en position cadrative, en tête de la deuxième proposition (16).

(14) "En Allemagne, les choses vont de mal en pis pour Helen. Kurt lui enlève son fils Johannes. Jack découvre que Caroline continue à prendre de l'hérö̈ne. Au Pakistan, Roquia essaie d'arrêter un important trafiquant. Mais celui-ci verse des pots de vin au juge..." (Traffik-Le sang du pavot)

(15) "Le jeune médecin Pierre de Sérizy se promène en forêt avec sa femme, Séverine. Il lui reproche d'être trop froide. Il la fait ligoter au pied d'un arbre puis fouetter. Elle a imaginé cela... Le couple fait chambre commune et lit séparé. Pour l'anniversaire de leur mariage, Pierre offre à Séverine un séjour aux sports d'hiver. Un homme cynique, Henri Husson, la poursuit de ses assiduités. Elle apprend de la maîtresse d'Husson, l'existence, dans un coin de Paris, d'une maison de rendez-vous tenue par Madame Anaïs, où les hommes viennent assouvir leurs fantasmes sexuels. Fascinée, Sandrine s'y rend. Sous le nom de "Belle de jour", elle devient une habituée." (Belle de jour)

(16) "A la veille de la rentrée des classes en septembre 1963, Anne, 13 ans, qui rentre en 4 è et Frédérique, 15 ans, qui rentre elle en seconde, quittent leur père pour rejoindre leur mère qui vit à Paris. Au lycée Jules Ferry, grouillant de jeunes filles, c'est d'abord la ronde des professeurs qui justifie leurs petites manies, puis ce sont les cours, le chahut et les amitiés. En dehors du lycée, Anne et Frédérique tentent de se satisfaire d'une vie familiale difficile car leur mère, divorcée, assume maladroitement son rôle d'éducatrice." (Diabolo menthe)

Au-delà de ces quelques caractéristiques, ces textes présentent aussi une certaine variabilité, notamment, pour ce qui nous intéresse, dans la forme des adverbiaux détachés en tête de phrase. C'est cette variabilité dans une structure relativement stable que nous allons observer.

\section{Examen des indices de fin de portée des cadres spatiaux dans les résumés de films}

\subsection{Premier bilan}

Cette question de la portée des adverbiaux a donné lieu à plusieurs travaux, dont une grande partie est consacrée aux cadres temporels. Dans ce domaine, 3 indices principaux ont été retenus (Le Draoulec \& Pery-Woodley 2001, 2003), dans une optique de traitement automatique :

- Apparition d'une expression temporelle référant à un temps qui n'est pas inclus dans la période dénotée par l'introducteur de cadre.

\footnotetext{
${ }^{4}$ http://www.ifrance.com/x-philes/saison1.htm
} 
- Changement de temps verbal (en particulier, passage au présent)

- Changement de paragraphe

Nous allons examiner si ces critères sont valables pour repérer la fin de portée des cadres spatiaux dans notre corpus. Le dernier est d'ores et déjà exclu car aucun texte ne présente d'alinéa.

Le premier critère reste pertinent dans le domaine spatial. L'apparition d'une expression spatiale référant à un espace qui n'est pas inclus dans l'espace dénoté par l'introducteur de cadre clôture le cadre en cours. C'était le cas dans l'exemple (14) où l'apparition de l'expression Au Pakistan ferme le cadre précédent ouvert par En Allemagne. C'est également le cas en (16) où En dehors du lycée vient clore le cadre ouvert par Au lycée Jules Ferry.

Lorsque un nouveau référent spatial est introduit en position non cadrative, on infère assez facilement, d'après les exemples observés, qu'il reste inclus dans le cadre en cours. Plus exactement, nous n'avons pas trouvé de cas, dans le corpus examiné, où un référent spatial introduit en position non initiale vient clore un cadre ouvert par une expression initiale (sur 65 textes, cela ne signifie pas que ce soit impossible mais témoigne néanmoins d'une tendance générale). Dans (17) et (18), une immense bibliothèque et la terrasse d'un café pourraient être indexés à d'autres lieux que Berlin et Toulouse respectivement. Mais il semble que le traitement naturel soit de rattacher, lorsque l'inclusion est possible, le nouveau lieu au cadre en cours, qu'il apparaisse en position focale d'une proposition principale, ou dans une subordonnée. Egalement, dans (19) on est amené à penser que le bal des croque-morts a lieu à Harlem et pas ailleurs.

(17) "Berlin. La ville est peuplée d'anges que seuls les enfants peuvent voir. Deux d'entre eux, Damiel et Cassiel, ont trouvé refuge dans une immense bibliothèque. Ils aimeraient aider les humains, murés dans leur solitude et leur silence, mais restent impuissants. Au hasard de ses errances, Damiel découvre Marion, trapéziste dans un petit cirque." (Les ailes du désir)

(18) "Juillet 1984. Bernard Thiraud, étudiant en histoire, part en vacances avec sa petite amie Claudine. A Toulouse, il consulte les archives de la préfecture. Alors qu'il rejoint Claudine à la terrasse d'un café, il est suivi par une voiture et abattu en pleine rue. C'est le jeune inspecteur Cadin qui est chargé de l'enquête. A Paris, il apprend que le père de Thiraud est mort en 1961 dans des circonstances semblables..." (Meurtres pour mémoire)

(19) "A Harlem, dans les années 50. Jackson, employé modèle d'une entreprise de pompes funèbres, résiste à toutes les tentations. Pur et dévot, il s'accorde cependant chaque année une distraction : le bal des croque-morts. C'est là qu'il fait la connaissance d'Isabelle, une splendide jeune femme dont il s'éprend éperdument." (Rage in Harlem)

En ce qui concerne le deuxième critère, qui repose sur le repérage de changements de temps, il est difficile de l'estimer à partir de notre corpus où le présent est prédominant. Nous avons cependant trouvé quelques rares cas, dont deux sont reportés en (20) et (21).

(20) " Vétéran du Viêt-nam, Joe Hoffman retourne au Laos pour retrouver sa maîtresse, Michelle Twassoon, disparue depuis treize ans. En chemin, il se lie d'amitié avec Charlie Pope, un commerçant ambulant. Joe est capturé par Larry Bingo, lui aussi ancien du Viêt-nam, et par sa troupe de déserteurs. // Pendant la guerre, Joe 
Hoffman avait dénoncé Larry Bingo à la suite du viol d'un enfant. Aujourd'hui, Bingo veut se venger." (Amère vengeance)

(21) "Un homme entre dans l'auberge de l'Abbaye, quelque part en pleine montagne. Il s'appelle Roland Brissot, il a la main gauche gantée et porte un coffret sous le bras. Un orage éclate, plongeant les lieux dans l'obscurité. Lorsque la lumière revient, le coffret a disparu. L'homme raconte son histoire. //Un an auparavant, il était un artiste raté. La femme qu'il aimait, une dénommée Irène, le méprisait." (La main du diable)

Dans ces exemples, un changement de temps apparaît dans la portée potentielle d'un cadre spatial. On note cependant que les décrochages temporels sont amorcés par un cadre temporel (pendant la guerre en (20) et Un an auparavant en (21)) dont on suspecte qu'ils sont attachés à un autre cadre spatial que celui en cours. Si ce critère des changements de temps n'est pas très probant, sur ce corpus, une autre piste a été d'observer si l'apparition d'un cadre temporel à la suite d'un cadre spatial est susceptible de fermer ce dernier.

\subsection{Combinatoire entre cadre spatial et cadre temporel}

Le corpus abonde en effet de cas où une proposition indexée par un cadre spatial est suivie d'une autre proposition indexée par un cadre temporel. Dans cette configuration, on infère ou non un changement de cadre spatial, sans que le nouveau cadre, s'il y a changement, soit spécifié. Mais les choses ne sont pas claires, car il est difficile de discerner si le changement d'espace inféré est déclenché simplement par l'apparition du cadre temporel, ou par d'autres indices convergents notamment la nature de la prédication (active/passive, télique, atélique etc.), qui pourrait être elle même l'indice de l'introduction d'une nouvelle relation de discours :

(22) Baltimore, trois personnes ont eu le foie arraché. A chaque fois, il persiste un mystère : l'impossibilité de déterminer par où est passé l'assassin. Mulder découvre que des affaires similaires ont eu lieu en 1903, 1933 et 1963, où à chaque fois il y a eu 5 victimes. A l'aide d'un ancien policier, Mulder découvre que le coupable est un mutant.

Est-ce que le «mystère persiste » à Baltimore ? Il semble que non, que l'on sorte du cadre pour entrer directement dans la problématique de l'enquête. On note, en outre, le passage du passé composé au présent, et l'usage d'une prédication impersonnelle qui réfère à un état n'ayant pas besoin d'être indexé spatialement. L'accent est mis sur la répétition dans le temps : d'autres affaires similaires ont eu lieu sans que l'on précise où.

Dans l'exemple suivant (23) :

(23) En Angleterre, un aristocrate prend feu devant sa femme. Quelques jours après, Mulder reçoit une cassette de son ancienne collègue et fiancée Phoebe Green, agent à Scotland Yard. Elle lui apprend également que l'incendiaire envoi des lettres romantiques aux femmes de ses victimes. Mulder parle d'un don de pyrokynésie, le pouvoir d'enflammer des objets à distance. Sir Malcolm arrive à Cape Code, Massachussetts, où il est accueilli par l'étrange Bob [...]

il ne semble pas que quelques jours après soit responsable de la fermeture du cadre. On pourrait avoir quelque chose comme, En Angleterre, un aristocrate prend feu devant sa femme. Quelques jours après, elle disparaît mystérieusement. Dans ce cas, le cadre temporel serait 
enchâssé dans le cadre spatial. Toute la description se situerait en Angleterre. En revanche, le lecteur est supposé savoir que Mulder enquête généralement aux Etats Unis et peut déduire de cette connaissance que le segment introduit par quelques jours après n'est plus indexé par En Angleterre.

(24) Dans une discothèque, un homme est abordé par une fille qui le séduit simplement en lui prenant la main. Après leurs ébats, le jeune homme meurt à la suite de la rupture d'une artère et la personne qui ressort de la salle de bain est en fait un homme. Mulder présente quatre autres décès similaires à Scully. Toutes les victimes, hommes et femmes, présentent un excès dephéromone [...]

Dans l'exemple (24), on a un adverbial temporel événementiel (Après leurs ébats). On infère assez facilement que leurs ébats n'ont pas eu lieu dans la discothèque mais que la soirée s'est poursuivie ailleurs, intuition attestée par l'introduction d'un nouveau référent spatial disjoint de la discothèque : la salle de bain.

(25) Dans un pénitencier, un détenu est témoin d'une expérience pratiquée par un médecin sur un autre détenu, John Barnett, déclaré décédé alors qu'il vit toujours. Quelques années plus tard, Mulder et Scully sont appelés sur les lieux d'un holdup dont le responsable semble être John Barnett. Un mauvais souvenir pour Mulder qui avait participé à son arrestation. Durant l'enquête, il s'aperçoit que le docteur Joe Ridley a fait des expériences sur le ralentissement de la vieillesse.

Dans l'exemple (25), contrairement aux exemples précédents, l'amplitude de l'intervalle temporel est large (quelques années plus tard). Cependant, cela ne semble pas avoir une incidence majeure pour décider si l'on sort du cadre spatial, en particulier d'un pénitencier où l'on peut très bien passer plusieurs années. On aurait pu avoir, Quelques années plus tard, la même chose se reproduit, le détenu est à nouveau témoin d'une étrange expérience. Or on a Mulder et Scully sont appelés sur les lieux d'un hold-up dont le responsable semble être John Barnett. Dans cette suite, l'introduction d'un nouveau référent spatial (les lieux du hold-up) a priori incompatible avec le pénitencier suggère un changement de cadre. Mais il ne semble pas, là non plus, que l'apparition du cadre temporel soit déclencheur de la fermeture du cadre spatial. C'est plutôt, la nature de la prédication, l'introduction d'un nouveau référent spatial incompatible avec le premier, et/ou l'introduction de nouveaux personnages qui en seraient responsables.

L'exemple (26) illustre un cas où toute la scène semble bien se passer dans le lieu fixé par l'adverbial en début de texte, au Mexique. On constate dans ce cas que l'apparition du cadre temporel ne déclenche pas systématiquement la fermeture du cadre spatial en amont,

(26) Au Mexique, un entomologiste employé par une grande entreprise pharmaceutique pour collecter de nouvelles espèces d'insectes à fort coefficient médical découvre sur une dépouille un insecte parasitant son hôte. Le soir même de sa découverte, le chercheur semble dans un état maladif grave...

On pourrait cependant être tenté de dire que lorsque l'amplitude du cadre temporel est très faible, comme ici, (le soir même de sa découverte), il y a de plus fortes chances pour que s'étende la portée du cadre spatial en amont. Mais, cela supposerait que l'on fait l'hypothèse première que chaque énoncé a besoin d'être indexé spatialement. Or, ça nous semble aller un peu vite en besogne. Nous examinons ce point dans la prochaine sous-section 3.3. 


\subsection{Portée et coïndiciation des événements}

Dans l'exemple (26), un énoncé d'état comme le chercheur semble dans un état maladif grave, n'a pas besoin d'être indexé spatialement. Il est simplement en relation avec ce qui précède (comme conséquence probable de sa découverte), et entre de ce fait dans la portée cadrative (cf. Charolles et Vigier, ce numéro) de l'adverbial spatial. Pour Gosselin (1990), en revanche « les phrases qui sont interprétées comme exprimant des événements supportent des compléments circonstanciels (p.39) ». Il discrimine les événements par la question Qu'arrive-t-il ?

(27) Qu'arrive-t-il?

Pierre est malade.

Il pleut.

?? L'homme est un animal bipède.

?? Pierre est le fils de Jean. (Gosselin, 1990 : 39)

(28) Pierre est épouvantablement malade, le soir, à Londres. (id.)

(29) Il pleut épouvantablement, le soir, à Londres. (id.)

(30) *L'homme est un animal bipède, le soir, à Londres. (id.)

(31) *Pierre est le fils de Jean, le soir, à Londres. (id.)

(32) Depuis la nuit des temps, l'homme est un animal bipède. (id.)

Selon L. Gosselin, tout événement suppose des circonstances de lieu, de temps, de cause et de manière. Au niveau du texte, il postule ainsi que l'on trouve des chaînes circonstancielles avec des catégories vides lorsque les circonstants ne sont pas réalisés. L'exemple (34), emprunté à Gosselin (1990 : 43) illustre une chaîne temporelle coréférentielle et une chaîne locative qui comporte des marques de décalage référentiel, des marques construites à partir d'un point de référence, comme le ferait une anaphore associative dans une chaîne référentielle.

(33) "[Il était à peu près huit heures du soir] i et [sur les eaux de la Marne], visible de la terrasse du restaurant Verjus, un brouillard épais commençait à régner (...). A droite $\left[d e e_{i}\right]$, la masse sombre des berges profilait de vagues contours impénétrables à la vue $\left[e_{i}\right]$; à gauche $\left[d e e_{i}\right]$, dans le ciel, il y avait un rougoiement perpétuel $\left[e_{i}\right]$ (...) » (P. Souvestre et M. Alain, 1987, p. 33, cité par L. Gosselin, 1990, p. 43-44).

Il conclut sa démonstration par ces propos :

«Nous pouvons donc affirmer que les circonstanciels de lieu et de temps réalisés syntaxiquement par des catégories vides sont nécessairement coïndicés avec des circonstanciels ou des marques de circonstances appartenant à d'autres phrases, sans que cette coïndiciation implique toujours la coréférence. Seul un changement de paragraphe ou a fortiori un changement de chapitre permet de suspendre provisoirement cette exigence. »

Cette vision des choses, issue de la tradition davidsonniene, mérite, selon nous, d'être nuancée. Il n'est pas certain en effet que le lecteur fasse toujours ce calcul de coïndiciation aux circonstances. Il semble même que dans de nombreux cas, on ne puisse faire hériter de façon certaine les circonstances introduites en amont aux énoncés subséquents, sans que pour autant cela gêne la lecture. Dans l'exemple suivant (34),

(34) "Dans un club de jazz, Greg Wonder, un saxophoniste de renom, dirige un excellent petit orchestre. Sur un terrain d'aviation, Eliot Vance virevolte aux commandes d'un ULM. // Tous les deux suspendent leur activité à la demande de mystérieux émissaires qui leur font une offre d'un million de dollars à charge, pour eux, de les suivre à New-York. Là, ils rencontrent un homme d'affaires... " (Attention les dégâts) 
le segment introduit par Tous les deux... n'a pas besoin d'être indexé spatialement. Cela impliquerait un calcul lourd où se poserait la question du choix entre une lecture distributive (chacun, là où il est, interrompt son activité) et une lecture collective (impliquant que les deux personnages se soient retrouvés entre temps quelque part). On fait, en réalité, très bien l'économie de ce calcul en comprenant ce segment comme une rupture par rapport aux activités respectives des deux personnages, et comme une transition pour introduire, en position focale, le lieu de l'action, New-York, repris ensuite en position initiale pour indexer possiblement, l'ensemble des actions à venir.

Ce type de texte contraste avec la description pure comme dans 1'extrait cité par Gosselin (33), où il est possible que le principe de propagation des circonstances marche assez bien, tant qu'on se cantonne aux adverbiaux spatio-temporels. Dans les résumés de films, où on a souvent l'introduction d'un décor (le cadre), de plusieurs personnages et d'une succession d'actions, ce principe de propagation ne semble pas pertinent, bien qu'on ait affaire, le plus souvent, à des événements. Une piste à approfondir serait de porter attention aux types de procès: les prédications intransitives (qui prototypiquement cumulent les traits -agentif, - télique, référentiel, mode irréel, passif etc.) seraient plus dégagées de l'indexation circonstancielle que les prédications transitives (qui prototypiquement cumulent les traits + agentifs, + télique, + référentiel, avec un objet + affecté, actif etc.) (cf. Hopper \& Thompson 1980). De plus, le repérage de décalages entre énoncés plus ou moins transitifs ou intransitifs peut être tenu comme indice d'un changement, notamment comme indice d'un passage du récit au commentaire (cf. Combettes 1992). Mais on peut se poser la question de savoir si un commentaire doit être intégré dans la portée cadrative d'un adverbial. Selon Charolles et Vigier (ce numéro), cela est tout à fait possible, un adverbial pouvant inclure dans sa portée cadrative un segment qui ne serait pas affecté par sa portée sémantique, pour peu que ce segment ajoute une information en rapport avec le cadre en cours et soit d'une façon ou d'une autre (relation de discours, relation lexicale) rattaché à la phrase en tête de laquelle figure l'adverbial, ou au topique de discours développé dans les phrases indexées par l'adverbial. Cette position offre une vision beaucoup plus large de la notion de portée qui permet de rendre compte des stratégies d'organisation des informations à communiquer et souligne, de ce point de vue, le rôle fonctionnel des adverbiaux. Elle permet en outre de rattacher des phrases sous la portée d'un adverbial sans pour autant que cela implique une relation de coïndiciation.

En conclusion de cette partie sur le repérage des indices de fin de portée, on voit qu'il n'est pas évident de trouver un critère fiable, ni même un faisceau de critères fiables. Le seul qui semble bien marcher, c'est celui de deux cadres incompatibles, mais cette configuration n'est pas si fréquente dans les résumés de films. Nous avons vu aussi que l'apparition d'un cadre temporel s'accompagne souvent de la fermeture du cadre spatial en cours, mais cela était plutôt dû à la nature de la prédication qui suivait qu'au cadre lui-même qu'il soit d'amplitude large ou étroite. Enfin, l'exploitation des contrastes entre énoncés +/- transitifs permet certes de repérer des segments de commentaires, mais la question reste encore à approfondir pour voir dans quelle mesure ces derniers peuvent être intégrés dans la portée cadrative d'un adverbial. Il faut noter, en outre, que les résumés de films emploient massivement le mode résultatif, condensé, où tous les événements sont présentés les uns après les autres souvent à la forme passive, tant dans la narration des événements que dans les passages de commentaires. La question reste donc à creuser plus amplement, et sur un corpus plus diversifié. Une chose remarquable cependant, c'est qu'il semble que bien souvent, on passe d'un espace à l'autre de façon diffuse, sans que cela soit marqué explicitement par des positions cadratives. Simplement, un espace est introduit puis des propositions arrivent dont on ne sait pas bien si elles entrent ou pas dans la portée de cet espace, 
puis un nouvel espace est introduit, pas nécessairement en position cadrative et on infère qu'il y a eu une évolution, un changement de lieu. C'est le cas dans l'exemple (34) déjà vu ainsi que dans l'exemple $(35)^{5}$ :

(35) "Sur une plage niçoise, en plein hiver. François Rollin, un auteur de télévision, fait la connaissance de Peggy, une jeune femme blonde et mystérieuse. ?//Elle lui affirme qu'elle est divorcée. Pour preuve, la présence chez elle de son avocat Marc Rilson. Ce dernier invite Rollin chez lui et lui demande de mettre fin à sa relation avec Peggy. Rilson va plus loin, en avouant que Peggy est sous le coup d'une inculpation pour meurtre ..." (Les seins de glace)

\section{4- Cadres ou forme d'ancrage}

Dans cette dernière partie, nous examinons comment la forme de l'adverbial en position frontale et son degré de détachement vis-à-vis de sa proposition d'accueil a une incidence sur son fonctionnement. La forme des adverbiaux et la façon dont ils sont détachés en tête de phrase sont en effet variables. On trouve des groupes prépositionnels détachés par une virgule ou suivis d'un point ou de deux points. On trouve aussi des noms propres, mis en exergue avec un effet de titre. $\mathrm{Au}$ fil des exemples commentés, nous nous attarderons en particulier sur certaines formes de détachement qui peuvent créer un « arrière-plan » servant à situer de manière diffuse une série d'événements, avec lesquels ils ne sont pas nécessairement coïndicés et dont ils ne constituent pas l'à propos.

Dans l'exemple repris en (36) ci-dessous, nous avons deux adverbiaux sous la forme de groupes prépositionnels détachés par une virgule. Ces deux adverbiaux définissent des cadres, l'apparition du second mettant fin à la portée du premier. Le premier cadre, indexé par en Allemagne, comprend une suite de propositions (les deux premières puis la troisième) qui n'ont d'autres liens entre elles que de se passer en Allemagne. Nous avons ensuite l'introduction d'un nouveau cadre, Au pakistan suivi d'une suite de deux propositions dont le topique est le personnage Roquia, en position sujet, repris par un démonstratif dans la seconde proposition.

(36) "En Allemagne, les choses vont de mal en pis pour Helen. Kurt lui enlève son fils Johannes. Jack découvre que Caroline continue à prendre de l'héroïne. Au Pakistan, Roquia essaie d'arrêter un important trafiquant. Mais celui-ci verse des pots de vin au juge..." (Traffik-Le sang du pavot)

Compte tenu du mode d'introduction des lieux et des personnages, on suppose qu'il s'agit du résumé d'un épisode d'un feuilleton. Le rédacteur fait le choix de rendre compte des activités des différents protagonistes selon leur situation spatiale, en Allemagne ou au Pakistan. Si l'on se réfère à la position de (Charolles 2003), En Allemagne serait topical (reliant les segments P1-P2 et P3 qui n'ont aucun autre lien que de se passer en Allemagne, alors qu'Au Pakistan, serait simplement cadratif, la fonction topicale étant assumée par le personnage Roquia, les deux propositions étant de plus dans une relation de Contraste. Mais le parallélisme de structure, entre les deux cadres peut nous inciter à leur attribuer une fonction semblable. Dans cette optique, on peut se questionner sur le statut réellement topical de En Allemagne, au sens où il ne constitue pas vraiment l'à propos des prédications subséquentes. Il ne s'agit pas en effet de ce qui se passe en Allemagne, mais de ce qui arrive aux personnages supposés connus dans l'hypothèse où nous avons affaire à un feuilleton. Nous pensons que dans cet exemple, les deux adverbiaux spatiaux

\footnotetext{
${ }^{5}$ Nous renvoyons à l'article de Charolles (2003) pour un commentaire détaillé sur ce texte.
} 
fonctionnent comme des introducteurs de cadres et exercent leur pouvoir de façon typique en indexant, spatialement, les propositions à leur suite.

Cette analyse rejoint l'idée défendue par Charolles qu' «à partir du moment où une intrigue se met en place, le SP de lieu ne constitue plus l'à propos du texte, il passe au second plan pour laisser le devant de la scène aux personnages ». (Charolles, 2003: 43). Dans l'hypothèse où nous avons affaire à un feuilleton, c'est en effet l'intrigue et les personnages qui priment, même si dans le premier segment indexé par en Allemagne on ne voit pas de rapport entre P1-P2 et P3.

Dans l'exemple (37) ci-dessous, on a deux groupes prépositionnels détachés par une virgule, en tête de phrase, les deux référents spatiaux entretenant une relation d'inclusion. Ces adverbiaux semblent intégrer dans leur portée cadrative l'ensemble du texte. Dans une interprétation restreinte, on pourrait toutefois limiter cette portée seulement à la première proposition dans la mesure où l'énoncé subséquent (l'objet contient les restes...) décrit un état qui n'a pas besoin d'être coïndicé aux circonstances.

(37) [Sur le site archéologique de Teso Dos Bichos, en Amérique du Sud], des indiens Secona découvrent une urne funéraire Amaru. L'objet contient les restes d'une femme shaman. Bilac, le coordinateur des fouilles, prévient le docteur Roosevelt, le guide de l'expédition, qu'il vaudrait mieux laisser l'urne à sa place. Mais Roosevelt n'en tient pas compte...

Il semble néanmoins que si le rédacteur a choisi de détacher les adverbiaux en tête plutôt qu'à la fin de la première proposition, c'est pour leur faire jouer un rôle supplémentaire à celui de marqueur des circonstances. En effet, on voit en (37') que le déplacement en fin de phrase ne nuit pas à la compréhension du texte.

(37') Des indiens Secona découvrent une urne funéraire Amaru, [sur le site archéologique de Teso Dos Bichos, en Amérique du Sud]. L'objet contient les restes d'une femme shaman. Bilac, le coordinateur des fouilles, prévient le docteur Roosevelt, le guide de l'expédition, qu'il vaudrait mieux laisser l'urne à sa place. Mais Roosevelt n'en tient pas compte...

Il semble dès lors que le positionnement stratégique en début de texte permet, certes, d'indexer spatialement la première proposition ${ }^{6}$, mais il permet, en outre, d'amorcer le récit et de poser le décor de toute l'intrigue. Il est difficile, dans ce cas, de faire de l'adverbial un topique au sens de ce à propos de quoi il est pertinent de dire quelque chose, le propos développé n'est pas en effet au sujet des lieux évoqués. La notion de topique comme point de départ conviendrait mieux (Halliday 1985). En effet, on perçoit aisément que ces deux adverbiaux détachés en tête servent d'ancrage pour développer l'intrigue, donnant d'entrée une masse d'informations associées aux référents mentionnés se rapprochant ainsi des frames à la Fillmore (1982). Comme si, de plus, l'espace et le temps constituaient des catégories suffisamment universelles pour qu'on puisse y rattacher n'importe quel type d'information connu ou moins connu. Par ailleurs, si ces deux adverbiaux détachés en tête étaient intégrés dans une proposition indépendante, on pourrait être tentée d'établir une relation 'd'Arrière plan' entre cette proposition et la suite du texte.

(37') [Tout se passe sur le site archéologique de Teso Dos Bichos, en Amérique du Sud]. Des indiens Secona découvrent une urne funéraire Amaru. L'objet contient les restes d'une femme shaman. Bilac, le coordinateur des fouilles, prévient le

\footnotetext{
${ }^{6}$ Sans doute dans ce cas un effet de persistance par défaut permet d'englober tout le texte dans la portée des adverbiaux.
} 
docteur Roosevelt, le guide de l'expédition, qu'il vaudrait mieux laisser l'urne à sa place. Mais Roosevelt n'en tient pas compte...

Dans la définition d'Arrière-plan (Asher \& Lascarides 2003, Vieu \& Prévot 2004), le constituant d'arrière-plan (l'état) est une toile de fond sur laquelle les événements sont interprétés comme dans

(38) It was pouring with rain. Mary came home,

impliquant, sur le plan sémantique, un recouvrement temporel (overlap) entre les éventualités des deux constituants. Vieu et Prévot (2004) suggèrent dans une note, que dans la première phrase de cet exemple (38), en dépit d'un ancrage temporel explicite, on peut considérer qu'il y a un implicit temporal viewpoint associé à l'état décrit. Ils proposent de voir une relation d'Arrièreplan $^{7}$ entre cet ancrage temporel implicite et la proposition qui suit, de la même façon qu'entre l'adverbial temporel et la proposition qui suit dans (39)

(39) That morning, the sun was shining.

La parenté avec les cadres de discours ne leur échappe pas et ils remarquent très justement que la portée de l'adverbial s'étend à tout le discours en (40) :

(40) That morning, the sun was shining. The spring atmosphere was very appealing. Lea decided to go for a walk. She put on her trekking shoes.

On peut dès lors prudemment émettre l'hypothèse qu'un adverbial spatial antéposé entretient avec les constituants compris dans sa portée une relation de discours Arrière-plan. Mais cette hypothèse mériterait qu'on y consacre une étude complète, l'espace et le temps n'impliquant pas les mêmes présupposés. Par ailleurs, dans un article récent (Vieu et alii, 2005 à par.), les phénomènes de portée large sont représentés dans la position topique. Mais les adverbiaux traités dans cet article ont un comportement plus classique, indexant plusieurs propositions et jouant un grand rôle sur le plan organisationnel. Dans notre corpus de " résumés de films », cette fonction d'indexation organisationnelle est partiellement atténuée, nous semble-t-il, au profit d'un fonction d'amorçage du texte. Pour l'heure, nous retiendrons donc l'idée que l'adverbial sert ici d'ancrage permettant d'embrayer sur les événements.

Dans l'exemple (41), deux adverbiaux spatiaux et temporels sont détachés fortement par deux points. Cette configuration spatio-temporelle apparaît deux fois dans le texte. L'adverbial spatial reste le même dans les deux occurrences, il s'agit d'un nom propre sans préposition Prague. Il est suivi dans les deux cas par une date, la seconde, antérieure à la première, permet d'introduire un flash-back :

(41) "Prague 1871 : dans son palais, le comte Anton est victime d'une crise de paralysie. Son épouse, Livia, le croyant à l'agonie, choisit de lui confier son secret... Prague 1865 : dans la capitale tchèque occupée par les Autrichiens, Livia se fait épouser par le comte. Survient un officier autrichien, Werner Ruiz, l'homme le plus débauché de tout Prague. Plus ou moins consciemment, Livia fait en sorte de le rencontrer. elle découvre bientôt la violence de l'amour..." (Senso)

Du fait de ce décalage temporel, on ne peut établir un vrai parallèle entre les deux cadres. Le deuxième, qui annonce le flash-back, serait l'élaboration d'un événement du premier cadre — en

\footnotetext{
${ }^{7}$ Background1 où l'événement précède l'état comme dans Mary came home. It was pouring with rain. Cette relation est distinguée de Background2 où l'état précède l'événement.
} 
1871, Livia confie un secret à son époux agonisant : en 1865, alors qu'elle venait de l'épouser, elle a rencontré un officier et en est tombée amoureuse... La mise en parallèle stratégique des deux introducteurs de cadres favorise cette interprétation de flash-back : l'espace reste constant, on est à Prague mais on se projette dans le passé, de 1871 à 1865, six ans plus tôt. Par ailleurs, on note qu'à l'intérieur de cette structure partagée sur le plan temporel, un contraste spatial plus précis se dégage : en effet deux adverbiaux spatiaux viennent à la suite des adverbiaux spatiotemporels. Ils sont eux même détachés en tête de proposition par une virgule. Ainsi on comprend qu'à deux époques différentes, dans la même ville, le premier cadre est restreint à l'espace intimiste du palais du comte mourrant, alors que dans le deuxième cadre, le contexte est élargi d'emblée : dans la capitale tchèque occupée par les Autrichiens. C'est dans cette situation historique particulière que vont survenir plusieurs événements dont la rencontre de Livia avec Werner Ruiz... Sur le plan spatial, on a comme un effet d'entonnoir, où la mention de la ville Prague sert d'amorce pour introduire à des époques différentes deux séries d'événements. Cette mention sert de toile de fond pour amorcer le récit des événements dans des sous-espaces différents (le palais et la ville occupée). Ce texte nous donne ainsi une illustration de cadres spatiaux et temporels (Prague + date) qui sont en relation Parallèle au niveau énonciatif et qui sont en relation Élaboration au niveau des événements dénotés.

Nous poursuivons l'examen d'une série d'exemples où les adverbiaux sont détachés en tête par une ponctuation forte: un point dans les exemples 42 à 45. Dans l'exemple (42), on a, comme dans le cas précédent, deux adverbiaux, un spatial et un temporel, sous la forme de groupes prépositionnels.

(42) "A Harlem, dans les années 50. Jackson, employé modèle d'une entreprise de pompes funèbres, résiste à toutes les tentations. Pur et dévot, il s'accorde cependant chaque année une distraction : le bal des croque-morts. C'est là qu'il fait la connaissance d'Isabelle, une splendide jeune femme dont il s'éprend éperdument." (Rage in Harlem).

Dans cet exemple, le détachement fort renforce l'impression déjà observée plus haut que contrairement à un cadre de discours où tous les événements sont coïndicés, on a affaire ici à un cadre qui permet, certes de situer l'action, mais surtout d'amorcer le récit. Ce procédé d'amorce semble être tout à fait typique des résumés de films et ressort d'autant plus que l'adverbial est détaché par une ponctuation forte : on donne un cadre spatial (et/ou temporel) puis on enchaîne sur les événements, en commençant par l'introduction d'un personnage en position topicale sujet dont on développe les aventures dans la suite. La présence des prépositions dans les groupes adverbiaux en (42) ménage toutefois un lien suffisamment fort avec les propositions subséquentes, pour être repris par le pronom adverbial là dans la clivée en position focale. Plus précisément le pronom reprend le bal des croque-morts, mais on infère facilement que c'est là, au bal des croque-morts, à Harlem, dans les années 1950, que Jackson fait sa rencontre. Ainsi, on a une structure où tous les événements ne sont pas coïndicés strictement aux circonstances, mais où les adverbiaux détachés en tête constituent une toile de fond (i.e. un arrière-plan) sur laquelle peuvent s'inscrire une série d'événements.

Dans (43), la configuration est un peu différente. L'adverbial est constitué d'un nom propre nu, Berlin. Puis, au lieu d'enchaîner directement sur l'introduction d'un personnage et de ses aventures, c'est « la ville » (anaphore) qui est topicalisée dans la première proposition. "Berlin" sert donc ici encore d'ancrage et de toile de fond, puis la proposition "la ville est peuplée d'anges..." fait la transition et permet d'introduire par la voix passive le thème du discours les anges qui sont nommés en position sujet de la proposition suivante. On pourrait être tentée de 
faire de Berlin dans cet exemple, un topique de discours, mais dès la deuxième proposition, le projecteur est mis sur les personnages et la ville repasse en arrière-plan comme toile de fond pour le récit.

(43) "Berlin. La ville est peuplée d'anges que seuls les enfants peuvent voir. Deux d'entre eux, Damiel et Cassiel, ont trouvé refuge dans une immense bibliothèque. Ils aimeraient aider les humains, murés dans leur solitude et leur silence, mais restent impuissants. Au hasard de ses errances, Damiel découvre Marion, trapéziste dans un petit cirque." (Les ailes du désir)

Dans (44), l'adverbial détaché en tête par un point Gare Montparnasse sert strictement d'ancrage au récit. Il situe en effet l'action du début du film, spatialement et temporellement (i.e. de nos jours) et même du tout début du film puisque dès la première proposition, la brésilienne attrape son train et quitte donc la gare. On a ici l'illustration parfaite de l'adverbial dont le rôle n'est pas tant d'indexer une série d'événements mais constitue simplement un moyen d'embrayer sur un événement.

(44) "Gare Montparnasse. Une ravissante Brésilienne attrape son train in extremis. Hélas, elle ignorait qu'en France, on composte les billets. Le contrôleur qui ne comprend rien au portugais, est bien décidé à la verbaliser. Par bonheur, une aimable avocate s'interpose et lui sert d'interprète. Après avoir sympathisé, les deux femmes se rendent au tribunal de Baugé où l'affaire de Marcel Petigas va être instruite..." (Maine Océan).

\section{Conclusion}

Nous avons montré au fil de ces exemples un fonctionnement particulier des adverbiaux spatiaux détachés en tête qui semble être très spécifique aux résumés de films. Trois traits importants se dégagent de notre analyse :

Tout d'abord, nous avons vu que les adverbiaux servent certes à regrouper des informations en créant des blocs mais que l'ensemble des propositions ainsi regroupées n'est pas forcément coïndicé aux circonstances fixées par l'adverbial. Cette observation rejoint la notion de portée cadrative développée dans ce numéro (cf. Charolles et Vigier) et va sans doute plus loin dans la mesure où se sont des propositions indépendantes (souvent des états) qui seraient ainsi être inclues dans la portée cadrative.

Le deuxième trait important qui se dégage de notre analyse est que conformément à l'analyse de Prévot (2003) et Chafe (1976), les adverbiaux spatiaux accèderaient difficilement au statut de topique. Seul l'exemple (43) installe pour un temps bref Berlin comme topique et cela est marqué par une reprise anaphorique en position sujet la ville. Sans pour autant rejeter l'idée que dans bien des cas, ils confèrent une cohérence a minima à une suite de propositions et interviennent de ce fait dans l'organisation des contenus propositionnels, ils ne constituent néanmoins pas l'à propos des propositions subséquentes, au sens de ce à propos de quoi il est pertinent d'énoncer quelque chose.

C'est plutôt la notion d'ancrage et d'amorce du récit qui les caractériserait le mieux ici. Ce troisième trait semble être le plus spécifique aux résumés de films, comme si le genre exigeait qu'on pose un décor de la façon la plus économique possible (i.e. la plus courte) avant d'introduire le ou les personnage(s) et l'intrigue. Nous avons vu que cette fonction d'amorce va parfois de pair avec la fonction cadrative au sens où l'adverbial sert en même temps à ancrer les personnages et les situations qui vont être introduits mais aussi à indexer, classiquement, une partie ou la totalité des propositions à sa suite. Nous avons également vu un cas extrême, 
l'exemple (44), où l'adverbial détaché fortement par un point ne peut servir que d'amorce au récit sans indexer la suite du discours, du fait de la sémantique du prédicat dans la proposition subséquente. Enfin, nous avons émit l'hypothèse que les adverbiaux ainsi détachés (plus particulièrement ceux qui sont détachés par une ponctuation forte et qui sont introduits sans préposition créant une sorte d'effet de titre ou de rubrique) pourraient entretenir avec les constituants entrant sous leur portée une relation Arrière-Plan, hypothèse qui devra, elle aussi être reconsidérée dans des travaux à venir, les introducteurs de cadre ayant par ailleurs été formalisé comme des topiques en SDRT dans un article récent (Vieu \& alii 2005, à par). Cette question reste ouverte pour de futures recherches. Elle suggère que selon le type d'adverbial et selon le genre de texte, nous n'aurions pas affaire à un fonctionnement unifié de ces marqueurs et qu'en y portant plus finement attention des différentiations et des nuances devraient émerger plus nettement. Leur rôle organisationnel pourraient être plus ou moins saillant au profit d'autres fonctions textuelles, comme celle d'amorçage par exemple, qui apparaît nettement dans les résumés de films.

\section{Références :}

Asher, N., and Lascarides, A. (2003). Logics of Conversation, Cambridge University Press.

Blinkenberg, A. (1969). Le problème de la transitivité en Français moderne. Munksgaard (ed), Hist. Filos. Medd. Dan. Vid. Selsk. 38, n¹ (1ére éd. 1960) kØebenhavn.

Borillo, A. (1992). "Le lexique de l'espace : prépositions et locutions prépositionnelles de lieu en français". Hommage à Nicolas Ruwet. L. Tasmovsky, A. Zribi-Hertz (eds), Ghent : 176190.

Borillo, A. (1993). «Préposition de lieu et anaphore », Langage 110, La couleur des prépositions C. Vandeloise (ed), 27-46.

Borillo, A. (1998). L'expression de l'espace en français. Ophrys, Paris.

Busquets J., Vieu L., Asher N. (2001). «La SDRT : une approche de la cohérence de discours dans la tradition de la sémantique dynamique » dans Cohérence et relation de discours à l'écrit, Verbum $n^{\circ} 23$. Péry-Woodley M.P. 2001 (ed), Presse Universitaire de Nancy, 73101.

Chafe W. L., (1976). «Givenness, contrastiveness, definiteness, subjects, topics and point of view », in Subject and Topics, New York Academic Press: 25-55.

Charolles M. (1993). "Les plans d'organisation du discours et leurs intéractions », dans Parcours linguistiques de discours spécialisés, Sciences pour la communication $n^{\circ} 41$. P. Lang (ed) Editions scientifiques européennes, Berne, 301-314.

Charolles M. (1994). "Cohésion, cohérence et pertinence du discours » dans Travaux de linguistique : La cohérence textuelle, cohésion et rupture. n²9. Editions Duculot, 125151.

Charolles M. (1997). L'encadrement du discours: univers, champs, domaines et espaces. In Cahier de Recherche Linguistique 6, Université Nancy 2. Rajouter l'adresse du site

Charolles M. (2003). « De la topicalité des adverbiaux détachés en tête de phrase ». Travaux de linguistique 47, Louvain : 11-49.

Charolles, M (à par.)

Charolles M., Le Draoulec, A., Pery-Woodley, M-P., Sarda, L. (2005, à par.). « Temporal and spatial dimensions of discourse organisation », JFLS, 15.2, Cambridge University Press.

Combettes B. (1992). L'organisation du texte, Coll. Didactique des textes, Université de Metz.

Cornish, F. (1998). Les 'chaînes topicales' : leur rôle dans la gestion et la structuration du discours. Cahiers de Grammaire 23 : 19-40. 
Filliettaz, L. (2001). Ch. 11 : L'organisation séquentielle et l'organisation compositionnelle. In Roulet, Filliettaz \& Grobet, 307-350.Foley W., Van Valin R. 1984 : Functional syntax and universal grammar, Cambridge studies in linguistics 38, Cambridge University Press.

Fillmore C. (1982). "Towards a descriptive framework for spatial deixis", in R. Jarvella \& E. Klein (eds), Speech, place and action, Londres.

Gaatone, D. (1976). "Locutions prépositives et groupes prépositionnels : observations sur la syntaxe de certains groupes prépositionnels." Linguistics 167: 15-34.

Gosselin L. (1990). «Les circonstanciels : de la phrase au texte »; Langue Françise 1990, n86. Larousse : 37-45.

Guimier C. (1993). 1001 circonstants, Presse Universitaire de Caen.

Halliday, M. A. K. (1985). An Introduction to Functional Grammar. London: Edward Arnold.

Halliday, M. A. K. and Hasan, R. (1976). Cohesion in English. London: Longman.

Hopper, P., Thompson S. (1980). "Transitivity in Grammar and Discourse." Language 56, 2: 251-297.

Le Draoulec A. \& Péry-Woodley M-P. (2001). "Corpus based identification of temporal organisation in discourse". In Rayson P., Wilson A., McEnery T., Hardue A., Khoja S. (eds) Proceedings of the Symposium on Corpus Linguistics 2001, Lancaster : 159-166.

Le Draoulec A., Péry-Woodley M.P. (2003). "Time travel in time: temporal framing in narratives and non-narratives", MAD'03.

Le Querler N. (1993). " Les circonstants en position initiale» dans 1001 circonstants, C. Guimier (ed), Presse Universitaire de Caen, 159-184.

Martin, R. (1983) La logique du sens, Paris, PUF.

Péry-Woodley M.P. (2001) (ed). Cohérence et relation de discours à l'écrit, Verbum $n^{\circ} 23$. Presse Universitaire de Nancy.

Prévost S. (2003). « Détachement et topicalisation : des niveaux d'analyse différents », Cahiers de Praxématique, $n^{\circ} 40$ : 97-126.

Prévost S. (2003). « Les compléments spatiaux : du topique au focus en passant par les cadres». Travaux de linguistique 47, Louvain : 51-77.

Reinhart, T. (1981). Pragmatics and linguistics: an analysis of sentence topics. Philosophica, 27: 53-94.

Sanders, T.J.M. and Spooren, W. (2001). Text representation as an interface between language and its users. In: T. Sanders, J. Schilperoord, and W. Spooren (eds), Text Representation Linguistic and psycholinguistic aspects . Amsterdam: John Benjamins.

Vieu, L \& Prévot L. (2004). « Background in SDRT », Workshop SDRT, TALN-04, Fès, 22 avril 2004.

Vieu L., Bras M., Asher N., Aurnague M. (2005 à par.) «Locating Adverbials in Discourse”, JFLS, 15.2, Cambridge University Press. 